\title{
Correction
}

Arq Neuropsiquiatr 2014;72(01):63-71

Insomnia: psychological and neurobiological aspects and non-pharmacological treatments

\section{Page 65}

Where it is written:

Intervention: cognitive therapy or cognitive reconstructuring

It should be:

Intervention: cognitive therapy or cognitive restructuring 